\title{
A CLINICAL STUDY OF BENIGN LESIONS OF PINNA
}

\author{
Srirangaprasad K1, Praveen Kumar², Shivanagouda Patil3, Ritesh Mahajan', Nagaraj T. M⿻5, George K. George ${ }^{6}$, Moby Boban $^{7}$ \\ 1 Professor, Department of ENT, Rajarajeshwari Medical College and Hospital, Mysore Road, Bangalore. \\ ${ }^{2}$ Associate Professor, Department of ENT, Rajarajeshwari Medical College and Hospital, Mysore Road, Bangalore. \\ ${ }^{3}$ ENT Consultant, Department of ENT, Rajarajeshwari Medical College and Hospital, Mysore Road, Bangalore. \\ ${ }^{4}$ Senior Resident, Department of ENT, Rajarajeshwari Medical College and Hospital, Mysore Road, Bangalore. \\ 5 Professor \& HOD, Department of ENT, Rajarajeshwari Medical College and Hospital, Mysore Road, Bangalore. \\ ${ }^{6}$ Post Graduate, Rajarajeshwari Medical College and Hospital, Mysore Road, Bangalore. \\ ${ }^{7}$ Post Graduate, Rajarajeshwari Medical College and Hospital, Mysore Road, Bangalore.
}

\section{ABSTRACT}

\section{BACKGROUND AND OBJECTIVES}

To study the clinical aspects of benign lesions of pinna and to evaluate aetiopathological factors, prevalence and management options.

\section{METHODS}

The study included 115 patients during the period from January 2013 to December 2013. The selection criteria included patients presenting with swellings of pinna which included keloids, seromas, sebaceous cyst, preauricular sinus, haemangioma, dermoid and neurofibroma. A detailed clinical history regarding onset, predisposing factors and associated conditions was documented and analysed.

\section{RESULTS}

Of total 115 cases in our study, 66 cases (57\%) presented with keloid followed by 33 cases (29\%) of pseudocyst of auricle, sebaceous cyst were seen in 10 cases, i.e. 9\% and only 1 case of Neurofibroma, Haemangioma and Dermoid was diagnosed ( $<1 \%) ; 94$ percent of patients presenting with Keloid were females; $88 \%$ of them had unilateral and just $12 \%$ had bilateral disease. Trauma was the factor in causation of pseudocyst of auricle in 11 cases (34\%), diabetes also was seen in 3 cases (9\%). Of all 33 cases of pseudocyst of auricle, 27 cases $(82 \%)$ were managed by window procedure and 6 cases $(18 \%)$ were managed with aspiration.

\section{CONCLUSION}

Trauma is the most important factor in causation of number of benign lesions of pinna. Other factors being high ear piercing through the cartilage. Diabetes mellitus plays a significant role and should be controlled simultaneously. Wide bore needle aspiration can be done for some of the cases of seroma patients who have recurrence and other cases can be managed by window procedure. Other lesions like keloid, sebaceous cyst, haemangioma and dermoid can be managed by complete surgical excision. A firm pressure bandage should be applied in most of the cases after surgery. Neurofibroma can be managed conservatively.

\section{KEYWORDS}

Keloid, Pinna, Preauricular Sinus, Benign Lesions, Trauma, External Ear.

HOW TO CITE THIS ARTICLE: Srirangaprasad K, Kumar P, Patil S, et al. A clinical study of benign lesions of pinna. J. Evolution Med. Dent. Sci. 2016;5(10):414-417, DOI: 10.14260/jemds/2016/95 \section{INTRODUCTION \\ Pinna contributes enormously to the facial aesthesis and is an} important part of peripheral auditory system. ${ }^{1}$ The peripheral auditory system functions to receive mechanical vibrations conduct these vibrations to the site of the primary receptor cells and thereby transduce this energy into an encoded electrical signal form, appropriate for conduction into and analysis by central nervous system. The Reception, Conduction and Transduction processes are strictly determined by structural and functional characteristics of this special receptor.

The ear functions as an early warning system by detecting and locating potentially threatening environmental sound.

Financial or Other, Competing Interest: None.

Submission 19-12-2015, Peer Review 20-12-2015,

Acceptance 21-01-2016, Published 03-02-2016.

Corresponding Author:

Dr. Srirangaprasad $K$,

C/o House No. 424, $7^{\text {th }}$ Cross, $1^{\text {st }}$ Block,

Jayanagar, Bangalore-560011.

E-mail: ranganitha@yahoo.co.in

DOI: 10.14260/jemds/2016/95
Keloid is a benign connective tissue hypertrophy characterized by smooth, pink, rounded scar-like tumour that invariably follows trauma or incision in the skin. It appears to be secondary to a defect in collagenase, which results in overgrowth of collagen as opposed to hypertrophied scar, which is composed of immature collagen that has failed to convert from tertiary to quaternary form. ${ }^{2}$ Pseudocyst or seroma is an uncommon asymptomatic, non-inflammatory swelling of pinna, characterized by endochondral cyst formation. First case of seroma was documented in mid 1800 and Hartmann was the first to report such a condition in the year 1846. Engel coined the term "Auricular Pseudocyst." Sebaceous cyst is a common benign cyst caused by blockage of draining ducts of sebaceous glands leading to cystic dilatation of the gland as a result of accumulation of sebum. Retention or epidermal cyst is relatively common around the auricle, especially in the postauricular sulcus and lobule because it is rich in sebaceous glands. Some may arise from the hair follicle. 4 Neurofibromas are circumscribed, but nonencapsulated neoplasms of the nervous system. They can arise in all peripheral nerve elements including Schwann's cells, neurons, fibroblasts and perineural cells. This may occur in 
isolation or as a part of Von Recklinghausen's syndrome. ${ }^{5}$ The aim of this clinical study is to ascertain various benign lesions of pinna presenting in the outpatient and to manage them with appropriate and timely intervention.

\section{MATERIALS AND METHODS}

This study was carried out at Rajarajeswari Medical College and Hospital, Bangalore. A total of 115 cases attending ENT OPD with swelling of the pinna were studied. A prospective study was carried out during the period January 2013 to December 2013. All patients attending Otolaryngology outpatient with benign lesions of pinna were counseled for inclusion. A total of 115 patients got listed with written informed consent and were studied Pre-structured questionnaire including sociodemographic profile about the risk factors leading to benign lesions of pinna, history taking and clinical examination were done. The Performa was designed based on the objectives of the study. It was pre-tested and used after modifications. The selection criteria included patients presenting with swellings of pinna. A detailed clinical history regarding onset, predisposing factors and associated conditions was documented. In addition to the routine blood and urine examination, blood sugar levels were measured in relevant cases. Surgery was carried out under local anesthesia in all of the cases after obtaining written consent and from parents/guardians in case of children.

\section{RESULTS}

A total of 115 cases who presented to the ENT OPD with complaint of ear swelling were examined and diagnosed clinically before subjecting them to minimum relevant investigations and managed with appropriate timely intervention under strict aseptic precautions. Most of the patients in our study were in the age group of 10 to 19 years, i.e. 46 patients constituting $40 \%$. Next common age group was 20 to 29 with 35 patients or $30 \%$; 71 of patients $(62 \%)$ in the study were females and 44 i.e. $38 \%$ were males. The male-tofemale ratio was found to be $1: 1.4 ; 58 \%$ presented with keloid followed by $29 \%$ of pseudocyst of auricle and $2.6 \%$ presented with pre-auricular cyst. Sebaceous cyst were seen in $9 \%$ of patients. Only 1 case each of Neurofibroma, Hemangioma and Dermoid were diagnosed $(<1 \%$ each).

Most of the cases presenting with Keloid were females, i.e. $94 \%$, only $6 \%$ were males. Keloid was unilateral in $88 \%$ and bilateral in $12 \%$. Ear piercing was the only predisposing factor seen in our studies; 62 cases (94\%) were managed with complete excision of the lesion followed by intralesional triamcinolone and 4 patients were instituted only intralesional triamcinolone.

Out of 33 cases of pseudocyst of auricle, $91 \%$ were males and $9 \%$ were females in our study. Predisposing factors like trauma and diabetes was seen in $34 \%$ and $9 \%$ of the patients, respectively; 6 patients underwent aspiration of fluid out of which 2 cases responded to the treatment; 13 cases which recurred after initial aspiration were treated with window procedure and $100 \%$ treatment results was seen. Trauma as a predisposing factors was seen in $10 \%$ of all cases and $57 \%$ had ear piercing as predisposing factor. Diabetes was observed in $3 \%$ of patients and no factors could be elicited $30 \%$. Aspiration of fluid was done in $5 \%$ of patients; 63 cases, i.e. $55 \%$ of the patients underwent complete excision of the lesion, $3 \%$ of the patients was treated by intralesional triamcinolone injections only and 1 case was managed conservatively.

\section{DISCUSSION}

Most of the patients in our study were young because young people are more concerned about their cosmetic appearance and since pinna is very important part of facial aesthesis, any lesions attracts their attention early. Another reason that could explain the high incidence in this active working age group is the hazards they encounter in their occupation. Socioeconomic status was based on modified Kuppuswamy scale. Here the education level, occupation of head of the family and per capita family income was taken into account. In our study $64 \%$ belonged to middle socioeconomic status, $5 \%$ were of upper class and the rest $30 \%$ belonged to lower class. This is because lesions of pinna-like keloid are quite common in middle and lower socioeconomic status, since poor hygienic conditions and aseptic ear piercing is widely practiced in this group. Also unhealthy social practices are not uncommon, which predispose to various lesions of pinna. Moreover illiteracy, lack of knowledge about asepsis and delayed seeking of medical assistance predisposes to more complication rates.

\section{Keloid}

The increased prevalence of Keloid could be attributed to increase in the "High piercing," i.e. ear piercing in the cartilaginous part of the pinna and ear lobule piercing which is considered fashion and traditional customs in society. This can be substantiated by a study by Christophfolz et al. on complications related to body piercing, male-to-female ratio was 1:2.2. Most of the recorded complications were related to the new vogue of piercing with the ear affected most commonly. The overall complication rate of ear piercing was found to be $35 \%$. High ear piercing accounted for most of the complications in their study. ${ }^{6}$ The reason for female preponderance is the custom of compulsory ear piercing by the females of our Indian society. Laterality of keloids depends on the site of ear piercing as well as on the genetic predilection of the individual to develop Keloid. In our study ear piercing was the only factor seen in causation of keloid.

In our study, 62 cases $94 \%$ were managed with complete excision of the lesion followed by intralesional triamcinolone acetonide $10 \mathrm{mg} / \mathrm{mL}, 0.5$ to $2 \mathrm{~mL}$, depending upon the length of the scar at monthly intervals for 4 months starting 2 weeks post-op as early institution may result in wound dehiscence; $6 \%$ of the patients which had no visible swelling, but Keloid could be palpated and found to $<5 \mathrm{~mm}$ in dimensions were instituted only intralesional triamcinolone in a dose mentioned above for 4 months. Patients were followed up for 6 months with no recurrences and no complications. Daniel J Rosen, et al. did a retrospective analysis of 64 patients representing 92 ear keloids. The treatment protocol consisted of excision with an intraoperative and two postoperative steroid injections. Success was achieved in $80 \%$ excised. ${ }^{7}$

\section{Pseudocyst of Auricle}

In our study trauma was the most common factor in causation of pseudocyst of auricle, seen in 11 cases (34\%), 3 cases (9\%) had diabetes mellitus which if uncontrolled can lead to delayed fluid resorption and in worst scenario may even cause perichondritis followed by destruction of auricular cartilage. Rest of the cases, $57 \%$ did not have any specific etiology. Our results are comparable with the study conducted by Kishore 
Chandra Prasad, Karthik S and Sampath Chandra Prasad, who with their experience of 116 cases of seroma found trauma as the leading predisposing factors accounting for 82 cases followed by insect bite in 13 and ear piercing in 21 cases. $^{1}$

In our study, 6 cases of pseudocyst of auricle were initially managed by aspiration under aseptic precautions followed by pressure bandage for 5 days with multivitamin and multiminerals supplementation. Two cases responded very well to aspiration and pressure bandage alone. Four cases who had recurrence following aspiration and also rest of the patients (27) were managed by window procedure with broad-spectrum antibiotic cover. Pressure dressing was applied in all cases. Following window procedure, all cases i.e. 31 cases $(100 \%)$ were treated successfully. Combined needle aspiration and pressure dressing on Pseudocyst with a short course of oral corticosteroids have an excellent result without recurrence. It has an additional advantage of being noninvasive modality of treatment. ${ }^{8}$

In a retrospective descriptive analysis by Lim et al. of pseudocyst of auricle, all 9 patients who had simple aspiration of the cyst had prompt re-accumulation of the pseudocyst. None of the patients had recurrence following excision and compression buttoning of the pseudocyst. The complication rate in study was $2.4 \%$. Only one patient developed initial perichondritis. ${ }^{9}$

\section{Sebaceous Cyst}

Ten cases of postauricular sebaceous cyst were included in our series and were managed with complete excision without any recurrences. It was in correlation with the study by Kishore Chandra Prasad, Karthik S and Sampath Chandra Prasad, who in their study of 39 cases of sebaceous cyst managed them by complete excision and did not observe any recurrence. ${ }^{1}$

\section{Hemangioma and Dermoid}

One case each of hemangioma and dermoid of pinna was observed in our study. This was managed with complete surgical excision.

\section{Neurofibroma}

Only 1 case of Neurofibroma of pinna was observed in our study. This patient had otitis externa and was managed by antibiotics.

\section{CONCLUSION}

The various benign lesions of pinna presenting to OPD in our study were found to be keloid, pseudocyst of auricle, preauricular sinus or cyst, sebaceous cyst, hemangioma, dermoid and neurofibroma of pinna, which present with swelling of the pinna with or without pain. Trauma is the most important factor in causation of number of benign lesions of pinna. Other factors are ear piercing as in keloids, diabetes mellitus plays a significant role in a few of the conditions and should be controlled simultaneously wide bore needle aspiration can be done for some of the cases of seroma. Patients who had recurrence can be managed by window procedure. Other lesions like keloid, sebaceous cyst, pre-auricular sinus or cyst can be managed by complete excision. Hemangioma and dermoid cases were managed by complete surgical excision with no recurrences and complications. A firm pressure bandage is to be applied in most of cases after surgery. Neurofibroma can be managed conservatively.

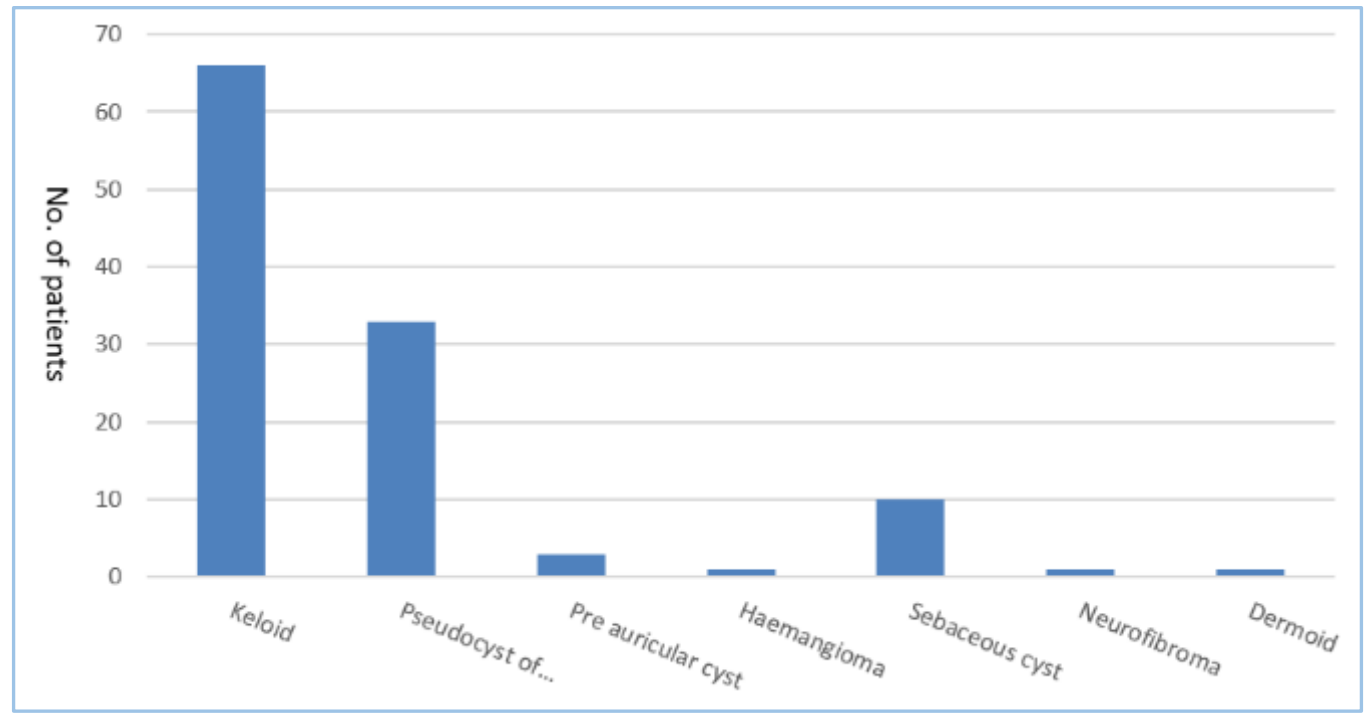

Fig 1: Case Distribution

\begin{tabular}{|c|c|c|c|c|}
\hline Diagnosis & Trauma & $\begin{array}{c}\text { Ear } \\
\text { Piercing/Iatrogenic }\end{array}$ & Diabetes Mellitus & Unknown \\
\hline Keloid & - & 66 & - & - \\
\hline Pseudocyst of auricle & 11 & - & 03 & 19 \\
\hline Sebaceous cyst & - & - & - & 01 \\
\hline Neurofibroma & - & - & - & 03 \\
\hline Pre-auricular sinus/cyst & - & - & - & 01 \\
\hline Haemangioma & - & - & - & 01 \\
\hline Dermoid & - & - & - & $\mathbf{3 5}(\mathbf{3 0} \%)$ \\
\hline Total & $\mathbf{1 1 ( 9 \% )}$ & $\mathbf{6 6 ( 5 7 \% )}$ & $\mathbf{0 3 ( 3 \% )}$ & \\
\hline
\end{tabular}




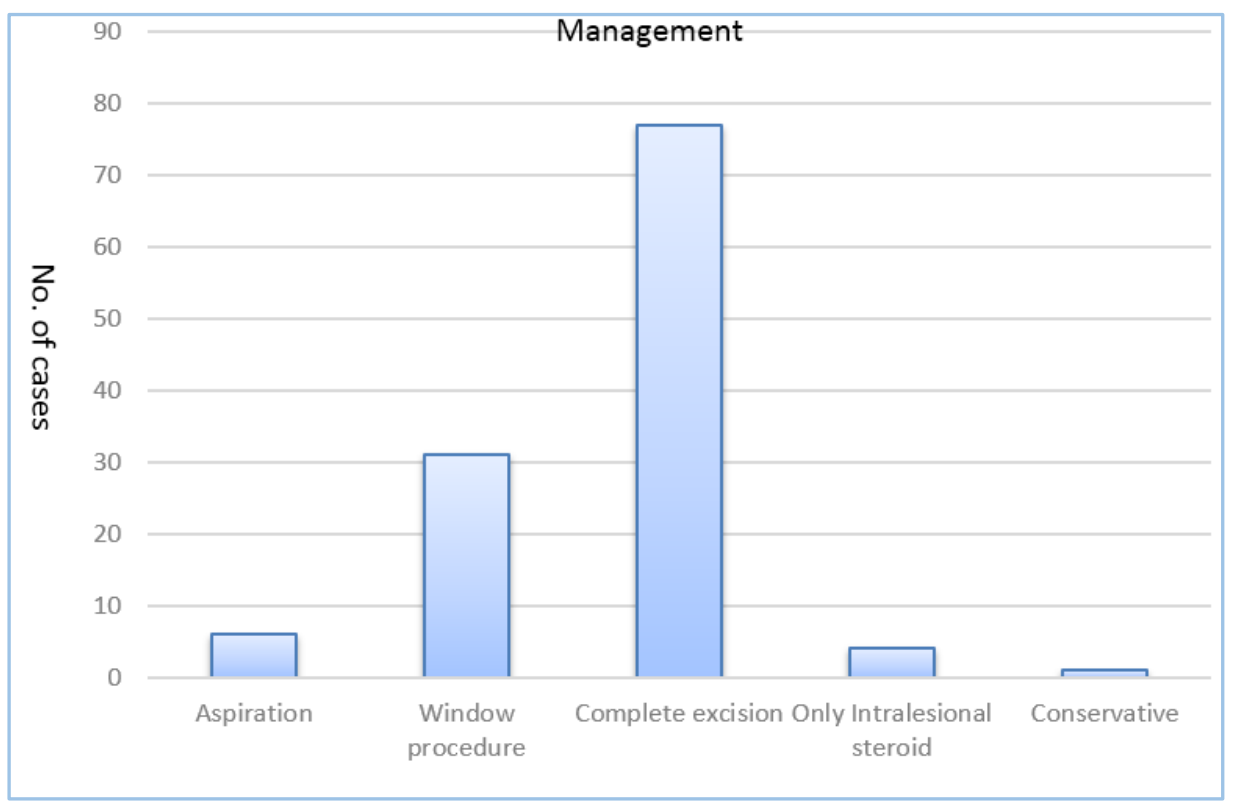

Fig 2: Treatment Options of all Cases

\section{BIBLIOGRAPHY}

1. Kishor Chandra Prasad, Karthik S and Sampath Chandra Prasad: A comprehensive study on lesions of pinna: American Journal of Otolaryngology-head and neck medicine and surgery (yearbook), 26(2005):1-6.

2. Masellis M, Ferrara MM. Extensive keloids in the auriclesurgical treatment by means of autologous grafts: Annals of Burns and Fire Disasters - vol. XII - n 4 - December 1999.

3. Engel D. Pseudocyst of auricle in Chinese. Arch Otolaryngol 1966;83:197-202.

4. Diseases of the external ear. Timothy TK Jung, MD, PhD; Tae Hoon Jinn, MD; Ballenger's Otorhinolaryngology; 16th edition; page 230-247.
5. Azhar M Shaida, Matthew W Young. Neurofibroma of pinna: ENT Journal 2007 Jan; 86(1):36-37.

6. Amy Han, Lian-Jie Li, Paradi Mirmirani. Successful treatment of auricular pseudocyst using a surgical bolster: a case report and review of the literature: Cutis. 2006 Feb; 77(2):102-4.

7. Daniel J Rosen, et al. A primary protocol for the management of ear keloids: results of excision combined with intraoperative and postoperative steroid injections: Plast Reconstr Surg. 2007 Oct; 120(5):1395-400.

8. Kaur S, Thami GP, Bhalla M. Pseudocyst of the auricle. Indian J Dermatol Venereol Leprol 2003;69:85-6.

9. Lim et al. Pseudocyst of the auricle: Laryngoscope. 2002 Nov; 112(11):2033-6. 